\title{
Novas ocorrências de hepáticas (Marchantiophyta) para o Estado da Bahia, Brasil ${ }^{1}$
}

\author{
Emilia de Brito Valente ${ }^{2,3}$ e Kátia Cavalcanti Pôrto ${ }^{2}$
}

Recebido em 15/12/2004. Aceito em 10/08/2005.

\begin{abstract}
RESUMO - (Novas ocorrências de hepáticas (Marchantiophyta) para o Estado da Bahia, Brasil). Na Serra da Jibóia, foram registradas 13 espécies de novas ocorrências, sendo oito para a região Nordeste: Bazzania aurescens Spruce, Calypogeia peruviana Nees \& Mont., Cephalozia crassifolia (Lindenb. \& Gottsche) Fulford, Lepidozia cupressina (Sw.) Lindenb., Pallavicinia lyellii (Hook.) S.F. Gray, Plagiochilla diversifolia Lindenb. \& Gottsche, Radula kegelli Gottsche ex Steph. e Riccardia fucoidea (Sw.) Schiffin., e cinco espécies referidas pela primeira vez para o Estado: Metzgeria albinea Spruce, Plagiochila gymnocalycina (Lehm. \& Lindenb.) Mont., P. simplex (Sw.) Lindenb., Riccardia amazonica (Spruce) S.W. Arnell e Symphyogyna aspera Steph. Para cada espécie são fornecidos comentários taxonômicos, ecológicos e distribuição geográfica mundial e no Brasil, além de indicação de literatura contendo descrição e ilustração. Foram realizadas ilustrações para algumas espécies.
\end{abstract}

Palavras-chave: Hepáticas, Mata Atlântica, região Nordeste

\begin{abstract}
New records of liverworts (Marchantiophyta) from Bahia State, Brazil). In Serra da Jibóia were recorded 13 species of new ocurrence wich are eight species from region Northeastern: Bazzania aurescens Spruce, Calypogeia peruviana Nees \& Mont., Cephalozia crassifolia (Lindenb. \& Gottsche) Fulford, Lepidozia cupressina (Sw.) Lindenb., Pallavicinia lyellii (Hook.) S.F. Gray, Plagiochilla diversifolia Lindenb. \& Gottsche, Radula kegelli Gottsche ex Steph. and Riccardia fucoidea (Sw.) Schiffin., and five are recorded for the first time from Bahia, State: Metzgeria albinea Spruce, Plagiochila gymnocalycina (Lehm. \& Lindenb.) Mont., P. simplex (Sw.) Lindenb., Riccardia amazonica (Spruce) S.W. Arnell and Symphyogyna aspera Steph. from Bahia, State. For each specie are provided taxonomics and ecological comments, geographical general and in Brazil distribution, and indication of literature with description and ilustration. Some species were ilustrated.
\end{abstract}

Key words: Liverworts, Atlantic Forest, region Northeast

\section{Introdução}

Foram catalogadas por Yano (1996) 1.125 espécies de hepáticas (Marchantiophyta) para o Brasil. Recentemente, Gradstein \& Costa (2003) reuniram em uma obra cerca de 600 espécies de hepáticas e antóceros para o País, citando em acréscimo cerca de 200 como duvidosas ou pouco conhecidas. Os referidos autores estimaram que o número de espécies desse grupo no Brasil deverá atingir cerca de 700-750 após a realização de levantamentos brioflorísticos em áreas ainda não exploradas.

Para o Estado da Bahia encontram-se citadas 124 espécies de hepáticas, compiladas dos catálogos de Yano $(1984 ; 1989 a ; 1995 ; 1996)$ e das seguintes publicações: Harley (1995) e Bastos et al. (1998b; 2000) para campos rupestres e mata de galeria em áreas do Parque Nacional da Chapada Diamantina; Vilas Bôas-Bastos \& Bastos (1998) para uma área de cerrado no município de Alagoinhas, BA; Bastos et al. (1998a) para diversas áreas de caatinga do Estado; C.J.P. Bastos (dados não publicados) para vegetação de restinga da região metropolitana de Salvador e do litoral norte do Estado, e Bastos \& Vilas Bôas-Bastos (2000a; b) e Bastos \& Yano $(2002$; 2003) que publicaram táxons de ocorrências novas e novos táxons para a ciência, respectivamente.

Este trabalho fornece o registro de 13 espécies de hepáticas de nova ocorrência para o Estado da Bahia, das quais oito ainda não haviam sido citadas para a região Nordeste. Todos os táxons foram coletados em um remanescente de Mata Atlântica, no Morro da Pioneira, zona norte da Serra da Jibóia, localizado no município de Santa Terezinha, interior da Bahia.

1 Parte da Dissertação de Mestrado da primeira Autora. Bolsista CNPq

2 Universidade Federal de Pernambuco, Centro de Ciências Biológicas, Departamento de Botânica, Av. Prof. Moraes Rego, 1235, CEP 50670-901, Recife, PE, Brasil

3 Autor para correspondência: ebvalente@bol.com.br 
O trabalho apresenta comentários taxonômicos e ecológicos, distribuição geográfica mundial e no Brasil; indicação de literatura contendo descrição, além de ilustrações para algumas espécies.

\section{Material e métodos}

A Serra da Jibóia $\left(12^{\circ} 51^{\prime} \mathrm{S}, 39^{\circ} 28^{\prime} \mathrm{W}\right)$ possui extensão de $6 \mathrm{~km}$. Caracteriza-se por apresentar trechos de caatinga na base, mata higrófila na encosta, e um afloramento rochoso de origem gnáissicogranítica no topo. A mata higrófila desenvolve-se nas encostas entre 400 e $800 \mathrm{~m}$ de altitude, caracterizando-se como Floresta Ombrófila Densa Montana de acordo com Veloso \& Góes Filho (1982).

Todas as amostras citadas no trabalho foram coletadas nas encostas da serra no interior da mata, em substratos variados, seguindo-se a metodologia usual para coleta e herborização de briófitas (Yano 1989b). As amostras encontram-se depositadas nos herbários da Universidade Federal de Pernambuco (UFP) e da Universidade Estadual de Feira de Santana (HUEFS). As identificações foram baseadas nos trabalhos de Bischler (1962), Fulford (1963; 1966; 1968), Hell (1969), Gradstein (1989), Gradstein et al. (2001), Reiner-Drehwald (1994), Heinrichs et al. (1998; 2000), Bernecker-Lücking (1999), Costa (1992), Lemos-Michel (2001) e Gradstein \& Costa (2003).

A distribuição geográfica foi feita seguindo-se Gradstein \& Costa (2003), e as siglas dos Estados brasileiros estão de acordo com o Instituto Brasileiro de Geografia e Estatística - IBGE.

\section{Resultados e discussão}

As 13 espécies de nova ocorrência pertencem às famílias Aneuraceae (duas espécies), Calypogeiaceae (1sp.), Cephaloziaceae (uma), Lepidoziaceae (duas), Metzgeriaceae (uma), Pallaviciniaceae (duas), Plagiochilaceae (três) e Radulaceae (uma). As espécies de nova ocorrência para o Estado da Bahia estão indicadas em asterisco (*) e as de nova ocorrência para a região Nordeste em dois asteriscos $(* *)$.

\section{MARCHANTIOPHYTA}

\section{ANEURACEAE}

* Riccardia amazonica (Spruce) S.W. Arnell, Svensk Bot. Tidskr. 56: 349. 1962.

\footnotetext{
Descrição: Hell (1969).
}

Ilustração: Hell (1969); Gradstein \& Costa (2003).

Material examinado: BRASIL. Bahia: Santa Terezinha, Serra da Jibóia, 2/XI/2001, Valente 147; ibid., 16/V/2004, Valente 367.

Comentários: Riccardia amazonica possui o eixo principal, em secção transversal, com quatro células de espessura e asas com duas células de largura. Androécio presente. Foi encontrada crescendo sobre tronco em decomposição no interior da mata.

Distribuição: Neotropical. No Brasil é encontrada nos Estados: AC, AM, AP, ES, PA, PE, RJ, RS e SP.

**Riccardia fucoidea (Sw.) Schiffn., Consp. Hepat. Arch. Ind.: 54. 1898.

Fig.1-7

Descrição: Hell (1969).

Material examinado: BRASIL. Bahia: Santa Terezinha, Serra da Jibóia, 15/XII/2003, Valente 256; ibid., 16/V/2004, Valente 369.

Comentários: Riccardia fucoidea possui gametófito dendróide, com ramificações opostas 3-pinadas, eixo principal sem asa, em secção transversal ca. 16 células de espessura, sendo as mais externas de coloração marrom e de paredes fortemente espessadas e ramificações (pinas) com asas de até 6 células de largura. Androécio presente. Foi encontrada sobre tronco em decomposição no interior da mata.

Distribuição: Neotropical. No Brasil é conhecida apenas para os Estados: RJ e SP.

\section{CALYPOGEIACEAE}

**Calypogeia peruviana Nees \& Mont. in Mont. Ann. Sci. Nat. Paris ser. 2, 9: 47. 1838.

Fig. 8-11

Descrição: Bischler (1962).

Material examinado: BRASIL. Bahia: Santa Terezinha, Serra da Jibóia, 2/XI/2001, Valente 129.

Comentários: a espécie apresenta os filídios com ápices inteiros ou com dois dentes, anfigastros distanciados com margens inteiras ou com dentes laterais largos e sinus arredondado. Encontrada em associação com Riccardia fucoidea sobre tronco em decomposição, no interior da mata.

Distribuição: América tropical. No Brasil é conhecida para os Estados: MG, RJ, RS, SP e SC.

\section{CEPHALOZIACEAE}

**Cephalozia crassifolia (Lindenb. \& Gottsche) Fulford, Mem. New York Bot. Gard. 11: 312. 1968.

Fig. 12-14 
Descrição: Fulford (1968).

Material examinado: BRASIL. Bahia: Santa Terezinha, Serra da Jibóia, 24/VIII/1996, Santos s.n. (HUEFS 52675).

Comentários: Cephalozia crassifolia é semelhante a Cephalozia crossi Spruce, diferenciando-se desta pelos filídios decurrentes, longitudinalmente inseridos e células medianas de 35-60 $\mu \mathrm{m}$ larg., enquanto que $C$. crossi apresenta filídios de base não decurrente, subtransversalmente inseridos, com células de 20-30 $\mu \mathrm{m}$ larg. O material examinado é semelhante aos sinônimos Cephalozia subforficata Herzog e Cephalozia caribbeania Fulford, em relação à abertura do ápice dos filídios. Foi encontrada em associação com Leucobrym martianum (Hornsch.) Hampe, Cyclolejeunea luteola (Spruce) Grolle, Odontoschisma falcifolium Steph. e Arachniopsis diacantha (Mont.) Howe, no interior da mata.

Distribuição: Neotropical. No Brasil ocorre nos Estados: ES, RJ e SP.

\section{LEPIDOZIACEAE}

**Bazzania aurescens Spruce, Trans. \& Proc. Bot. Soc. Edinburgh 15: 374. 1885.

Fig. 15-18

Descrição: Fulford (1968).

Material examinado: BRASIL. Bahia: Santa Terezinha, Serra da Jibóia, 2/XI/2001, Valente 158; ibid., 16/XII/2003, Valente 313.

Comentários: Bazzania aurescens apresenta os filídios predominantemente 3-denteados, sem vita; anfigastros clorofilados, tão largos quanto longos, irregularmente denteados no ápice e com base cuneada. Encontrada sobre tronco vivo, no interior da mata.

Distribuição: Neotropical. No Brasil ocorre nos Estados AM, GO, MG e SP.

**Lepidozia cupressina (Sw.) Lindenb. in G.L. \& N., Syn. Hepat.: 207. 1845.

Fig. 19-23

Descrição: Fulford (1966).

Material examinado: BRASIL. Bahia: Santa Terezinha, Serra da Jibóia, 2/XI/2001, Valente 156; ibid., 15/XII/2003, Valente 228; ibid., 16/XII/2003, Valente 312.

Comentários: Lepidozia cupressina apresenta gametófito com ramos flageliformes, filídios quadrífidos até $1 / 2$ a $1 / 3$ do comprimento, com os segmentos terminando em uma fileira de duas células e inseridos obliquamente no caulídio. Encontrada formando tufos sobre troncos vivos, no interior da mata. Segundo Gradstein \& Costa (2003), possivelmente L. brasiliensis Steph., que é uma espécie de larga distribuição no Brasil e de menor tamanho, é sinônimo de L. cupressina.

Distribuição: América tropical, África e oeste Europeu. No Brasil ocorre apenas nos Estados: RJ e SP.

\section{METZGERIACEAE}

* Metzgeria albinea Spruce, Bull. Soc. Bot. France 36 (Suppl.): 201. 1890.

Descrição: Costa (1992); Lemos-Michel (2001). Ilustração: Costa (1992); Gradstein \& Costa (2003).

Material examinado: BRASIL. Bahia: Santa Terezinha, Serra da Jibóia, 4/III/2001, Valente 54; ibid., 7/VII/2001, Valente 110; ibid., 16/V/2004, Valente 333.

Comentários: Metzgeria albinea tem a costa, em secção transversal, com duas fileiras de células em ambas as superfícies (dorsal e ventral), cerca de 10-15 células medulares e duas cerdas por célula marginal. Encontrada sobre tronco vivo, no interior da mata.

Distribuição: Pantropical. No Brasil é amplamente distribuída, ocorrendo nos Estados: AC, CE, ES, PE, PR, MG, RJ, RS, SC e SP.

\section{PALLAVICINIACEAE}

**Pallavicinia lyellii (Hook.) S.F. Gray, Nat. Arr. Brit. Pl. 1: 775.1821.

Descrição: Hell (1969).

Ilustração: Gradstein \& Costa (2003).

Material examinado: BRASIL. Bahia: Santa Terezinha, Serra da Jibóia, 16/XII/2003, Valente 316.

Comentários: Pallavicinia lyellii tem o talo, em secção transversal, com um cordão central, margem inteira e estrutura reprodutiva feminina protegida por escamas formando um invólucro. Ginoécio presente. Encontrada sobre tronco em decomposição, no interior da mata.

Distribuição: América Tropical, muito difundida nos paleotrópicos e regiões temperadas do leste da América do Norte e Europa. No Brasil é encontrada nos Estados: AC, AM, MS, PA, RJ, RS, SP e SC.

*Symphyogyna aspera Steph., in McCormick, Bot. Gaz. 58: 403. 1914.

Descrição: Hell (1969). 

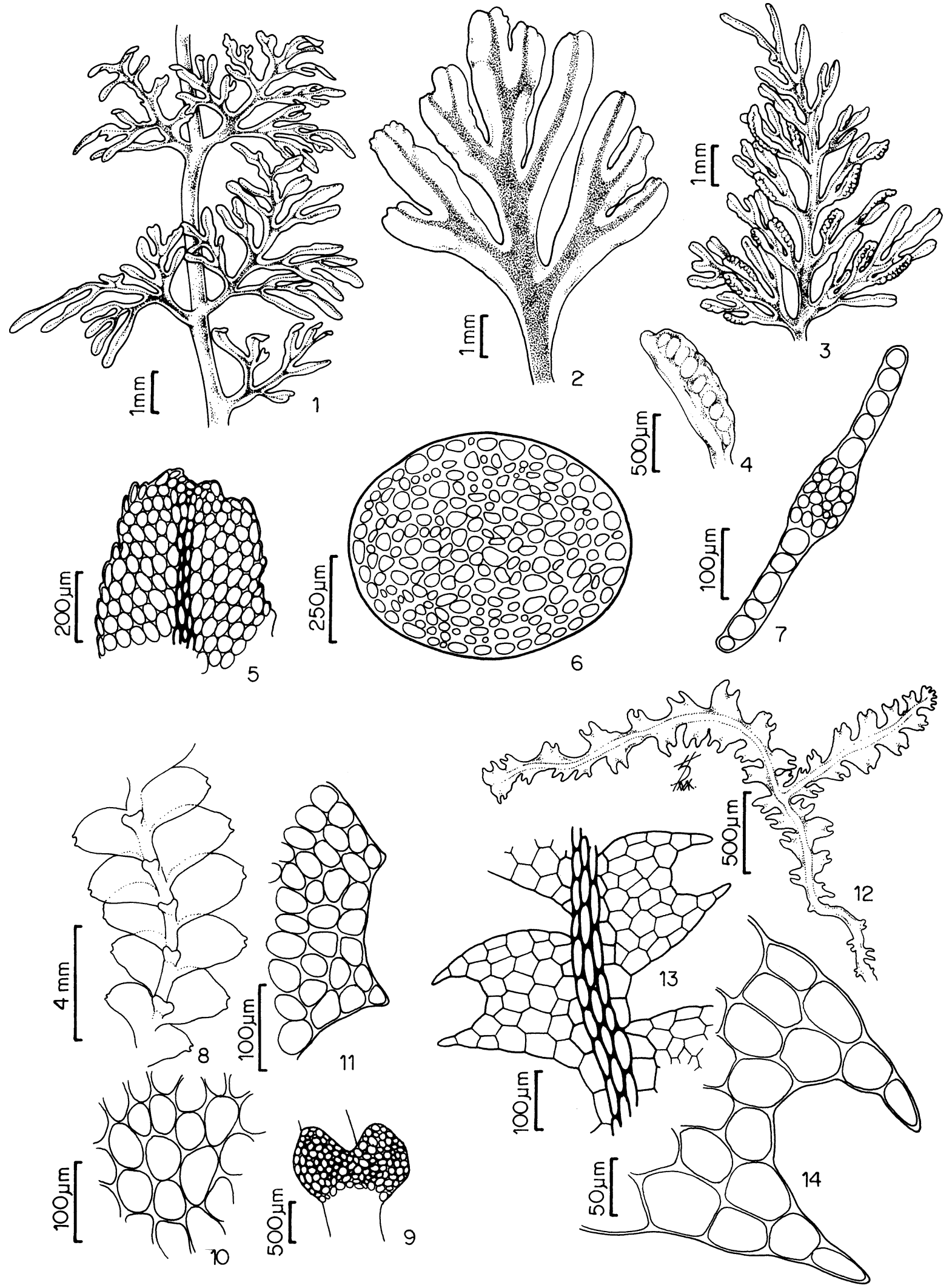

Figuras 1-7. Riccardia fucoidea (Sw.) Schiffn. 1.Aspecto geral do gametófito. 2. Pina 3. Ramo fértil com estruturas reprodutivas masculinas. 4. Detalhe do androécio. 5. Células do ápice de uma pina. 6. Secção transversal do ramo principal. 7. Secção transversal de um ramo secundário. Figuras 8-11. Calypogeia peruviana Nees \& Mont. 8. Aspecto geral do gametófito. 9. Anfigastro. 10. Células do filídio. 11. Células do ápice do filídio. Figuras 12-14. Cephalozia crassifolia (Lindenb. \& Gottsche) Fulford. 12. Aspecto geral do gametófito 13. Detalhe do ramo. 14. Células do ápice do filídio. 

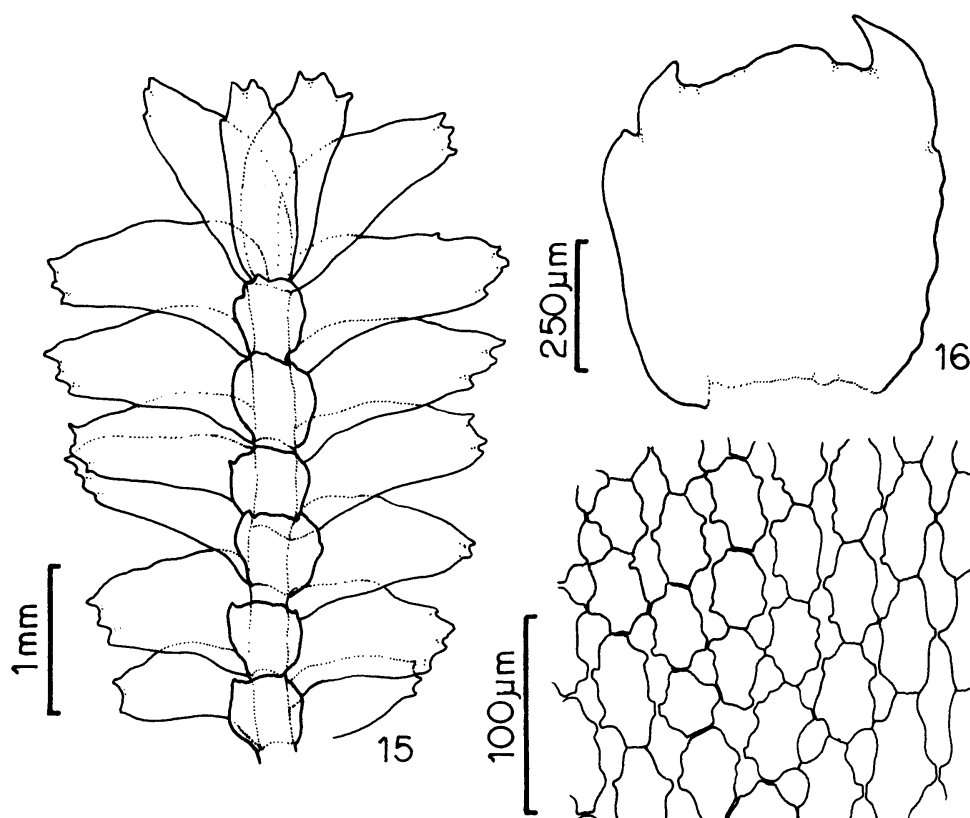

16
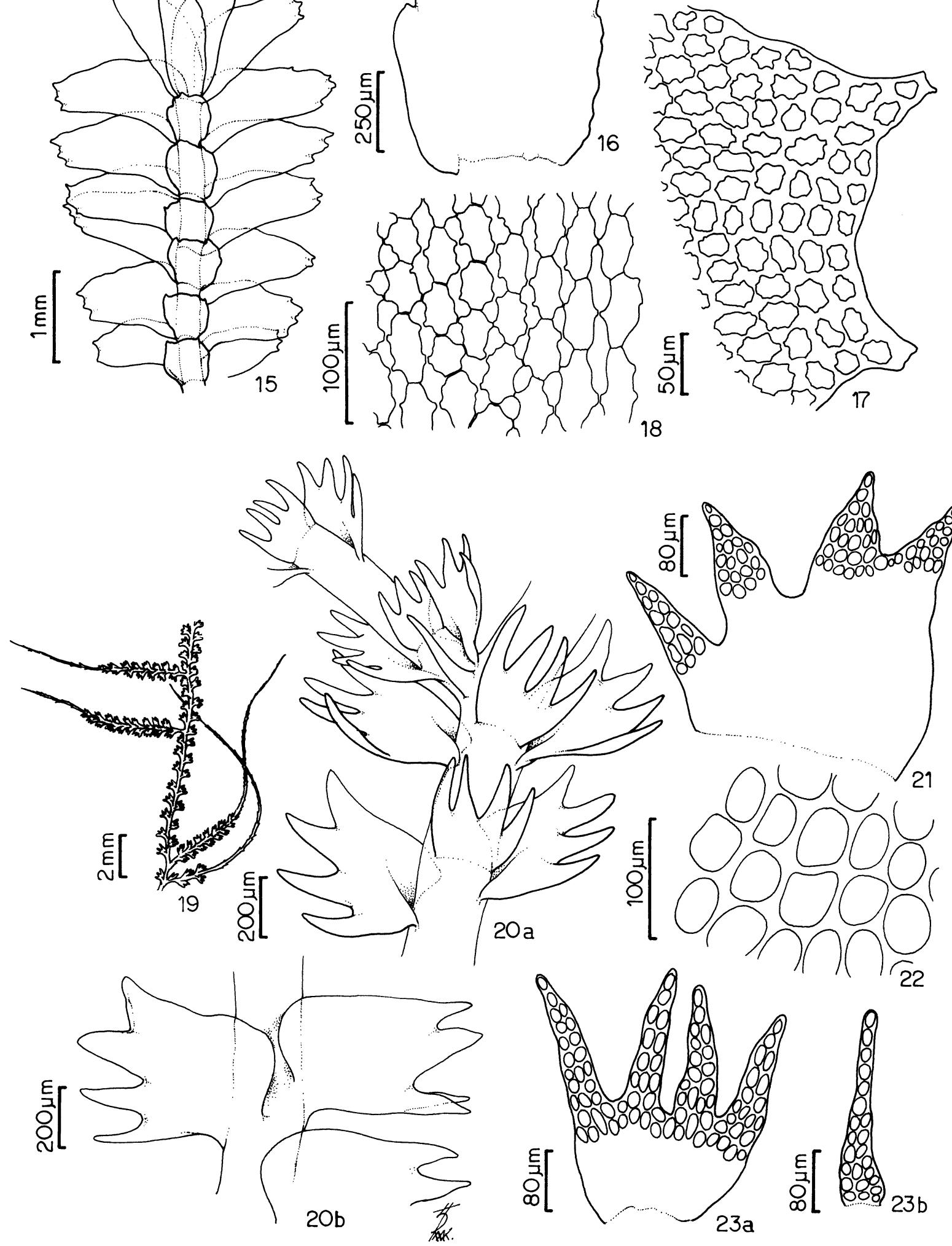

Figuras 15-18. Bazzania aurescens Spruce. 15. Aspecto geral do gametófito. 16. Anfigastro. 17. Células do ápice do filídio. 18. Células centrais do filídio. Figuras 19-23. Lepidozia cupressina (Sw.) Lindenb. 19. Aspecto geral do gametófito. 20a. Detalhe do ramo evidenciando a inserção do filídio, vista ventral. 20b. Detalhe do ramo evidenciando a inserção do filídio, vista dorsal 21. Filídio. 22. Células centrais do filídio. 23a. Anfigastro. 23b. Primeiro anfigastro de uma ramificação. 
Ilustração: Hell (1969); Gradstein \& Costa (2003).

Material examinado: BRASIL. Bahia: Santa Terezinha, Serra da Jibóia, 3/III/2001, Valente 28, ibid., Valente 37; 4/III/2001, Valente 67.

Comentários: Symphyogyna aspera possui o talo lobado (profunda ou superficialmente), com ramificações simples, e margens sem dentes, característica que a distingue de $S$. brongniartii Mont., que também possui talo lobado, mas apresenta dentes marginais. Encontrada sobre tronco em decomposição, no interior da mata.

Distribuição: Neotropical. Amplamente distribuída no Brasil: AM, ES, MG, PA, PE, RJ, RS, SC e SP.

\section{PLAGIOCHILACEAE}

**Plagiochila diversifolia Lindenb. \& Grottsche in Gottsche et al., Syn. Hepat.: 640. 1847.

Descrição e ilustração: Heinrichs et al. (2000).

Material examinado: BRASIL. Bahia: Santa Terezinha, Serra da Jibóia, 15/XII/2003, Valente 225; 16/XII/2003, Valente 314.

Comentários: Plagiochila diversifolia apresenta gametófito grande, 6-8 mm de larg., filídios com cerca de 15 dentes agudos, de 2-5 células de compr. e 1 célula de larg. até quase a base, distribuídos na margem superior, ápice, e até 1/3 da margem inferior da lâmina. Encontrada sobre tronco vivo e em decomposição, no interior da mata.

Distribuição: Neotropical. No Brasil ocorre apenas em AM, MG e RJ.

Plagiochila gymnocalycina (Lehm. \& Lindenb.) Mont., in d'Orbigny, Voy. Amer. Mér. 7, Bot. 2: 81. 1839.

Descrição: Heinrichs et al. (1998).

Ilustração: Heinrichs et al. (1998); Gradstein \& Costa (2003).

Material examinado: BRASIL. Bahia: Santa Terezinha, Serra da Jibóia, 15/XII/2003, Valente 244b; ibid. 3/XI/2001, Valente 176.

Comentários: Plagiochila gymnocalycina possui filídios ventrados e reprodução vegetativa por filídios caducos e fragmentados. Encontrada sobre tronco em decomposição no interior da mata.

Distribuição: Neotropical. No Brasil ocorre nos Estados: AC, MG, PE, RJ, SC e SP.

*Plagiochila simplex (Sw.) Lindenb., Sp. Hepat.: 54. 1840.

Descrição: Heinrichs et al. (1998).
Ilustração: Heinrichs et al. (1998); Gradstein \& Costa (2003).

Material examinado: BRASIL. Bahia: Santa Terezinha, Serra da Jibóia, 4/III/2001, Valente 47.

Comentários: espécie semelhante a $P$. gymnocalycina e $P$. subplana Lindenb., diferindo da primeira pelos filídios mais curtos, 1,5-2,5 x mais longos que largos, enquanto em $P$. gymnocalycina apresentam 2,5-5,0 x mais longos que largos; e, difere da segunda pelos filídios ventrados, que estão ausentes em P. subplana, e pelas células medianas da lâmina que em $P$. simplex medem 15-30 $\mu \mathrm{m}$ larg. e, em P. subplana, 30-45 $\mu \mathrm{m}$ larg. Androécio presente. Encontrada sobre tronco vivo, no interior da mata.

Distribuição: Neotropical. No Brasil ocorre nos Estados: AM, ES, MG, PE, PR, RJ, RS e SP.

\section{RADULACEAE}

**Radula kegelii Gottsche ex Steph., Hedwigia 23: 152. 1884.

Descrição: Reiner-Drehwald (1994).

Ilustração: Reiner-Drehwald (1994); LemosMichel (2001).

Material examinado: BRASIL. Bahia: Santa Terezinha, Serra da Jibóia, 15/XII/2003, Valente 235; ibid.16/XII/2003, Valente 388.

Comentários: Radula kegelii possui gametófito com ramificação dicotômica, filídios imbricados, porção basal do lóbulo cobrindo o caulídio, e quilha do lóbulo formando um ângulo de cerca de $90^{\circ}$ com o caulídio. Encontrada sobre tronco vivo no interior da mata.

Distribuição: Neotropical. No Brasil ocorre nos Estados: MT, PA, PR, RJ, RS, SC e SP.

\section{Referências bibliográficas}

Bastos, C.J.P. \& Vilas Bôas-Bastos, S.B. 2000a. Ocurrence of some Lejeuneaceae (Jungermaniophyta) in Bahia, Brazil. Tropical Bryology 18: 45-54.

Bastos, C.J.P. \& Vilas Bôas-Bastos, S.B. 2000b. Some new additions to the hepatic flora (Jungermaniophyta) for the state of Bahia Brazil. Tropical Bryology 18: 1-11.

Bastos, C.J.P. \& Yano, O. 2002. Pycnolejeunea porrectilobula (Lejeuneaceae), a new species from Brazil. Nova Hedwigia 74(3-4): 439-443.

Bastos, C.J.P. \& Yano, O. 2003. New records of the genus Rectolejeunea (Lejeuneaceae) for the state of Bahia, Brazil. Nova Hedwigia 76(3-4): 477-485.

Bastos C.J.P.; Albertos, B. \& Vilas Bôas, S.B. 1998a. Bryophytes from some caatinga areas in the State of Bahia (Brazil). Tropical Bryology 14: 69-75. 
Bastos C.J.P.; Stradmann, M.T. \& Vilas Bôas-Bastos, S.B. 1998b. Additional contribution to the bryophyte flora from Chapada Diamantina National Park, State of Bahia, Brazil. Tropical Bryology 15: 15-20.

Bastos, C.J.P.; Yano, O. \& Vilas Bôas-Bastos, S.B. 2000. Briófitas de campos rupestres da Chapada Diamantina, Estado da Bahia, Brasil. Revista Brasileira de Botânica 23(4): 357-368.

Bernecker-Lücking, A. 1999. Key to Latin American species of Bazzania S.F. Gray. Tropical Bryology 16: 117-126.

Bischler, H. 1962. The genus Calypogeja Raddi in South America II. Subgenus Calypogeja, subgroups 1, 2 and 3. Candollea 18: 53-93.

Costa, D.P. 1992. Hepáticas do Pico da Caledôea, Nova Friburgo, Rio de Janeiro, Brasil. Acta Botanica Brasilica 6(1): 3-39.

Fulford, M. 1963. Manual of Hepaticae of Latin America. Part I. Memoirs of the New York Botanical Garden 11(1): 1-172.

Fulford, M. 1966. Manual of Hepaticae of Latin America. Part II. Memoirs of the New York Botanical Garden 11(2): 173-276.

Fulford, M. 1968. Manual of Hepaticae of Latin America. Part III. Memoirs of the New York Botanical Garden 11(3): 277-392.

Gradstein, S.R. 1989. A key to the Hepaticae and Anthocerotae of Puerto Rico and Virgin Islands. Bryologist 92(3): 329-348.

Gradstein, S.R. \& Costa, D.P. 2003. The Hepaticae and Anthocerotae of Brazil. Memoirs of the New York Botanical Garden 87: 1-318.

Gradstein, S.R.; Churchill, S.P. \& Salazar Allen, N. 2001. Guide to the Bryophytes of Tropical America. Memoirs of the New York Botanical Garden 86: 1-577.

Harley, R.M. 1995. Bryophyta. Pp. 803-812. In: B.L. Stannard (ed.). Flora of the Pico das Almas. Chapada Diamantina - Bahia, Brazil. Kew, Royal Botanic Garden.
Heinrichs J.; Gradstein, S.R. \& Grolle, R. 1998. A revision of the neotropical species of Plagiochila (Dumort.) Dumort. (Hepaticae) described by Olof Swartz. The Journal of the Hattori Botanical Laboratory 85: 1-32.

Heinrichs J.; Anton, H.; Gradstein, S.R. \& Mues, R. 2000. Systematics of Plagiochila sect. Glaucescentes Carl (Hepaticae) from tropical America: a morphological and chemotaxonomical approach. Plant Systematic and Evolution 220: 115-138.

Hell, K.G. 1969. Briófitas talosas dos arredores da cidade de São Paulo (Brasil). Universidade de São Paulo. Boletim da Faculdade de Filosofia, Ciências e Letras, Botânica 25: $1-190$.

Lemos-Michel, E. 2001. Hepáticas epifíticas sobre o Pinheiro-Brasileiro no Rio Grande do Sul. Porto Alegre, Editora Universidade/UFRGS.

Reiner-Drehwald, M.E. 1994. El género Radula Dum. (Radulaceae, Hepaticae) en el Noreste da Argentina. Tropical Bryology 9: 5-22.

Vilas Bôas-Bastos, S.B. \& Bastos, C.J.P. 1998. Briófitas de uma área de cerrado no município de Alagoinhas, Bahia, Brasil. Tropical Bryology 15: 101-110

Yano, O. 1984. A checklist of Brazilian liverworts and hornworts. The Journal of the Hattori Botanical Laboratory 56: 481-548.

Yano, O. 1989a. An additional checklist of Brazilian the bryophytes. The Journal of the Hattori Botanical Laboratory 66: 371-434.

Yano, O. 1989b. Briófitas. Pp. 27-30. In: O. Fidalgo \& V.L.R. Bononi (eds.). Técnicas de coleta, preservação e herborização de material botânico. Série Documentos. São Paulo, Instituto de Botânica.

Yano, O. 1995. A new additional annotated checklist of Brazilian bryophytes. The Journal of the Hattori Botanical Laboratory 78: 137-182.

Yano, O. 1996. A checklist of the Brazilian bryophytes. Boletim do Instituto de Botânica 10: 47-232. 